\title{
Computer-Based Examples Designed to Encourage Optimal Example Processing: A Study Examining the Impact of Sequentially Presented, Subgoal-Oriented Worked Examples ${ }^{1}$
}

\author{
Robert K. Atkinson, \& Sharon J. Derry \\ Dept. of Counselor Education and Educational Psychology, Box 9727, Mississippi State, MS 39762 \\ Dept. of Educational Psychology, University of Wisconsin, 1025 W. Johnson St., Madison, WI 53706 \\ Tel: (662) 325-8178, (608) 263-3676, Fax: (662) 325-3263 \\ Email: atkinson@ra.msstate.edu, SharonD@wcer.wic.edu
}

\begin{abstract}
This study was designed to examine the effectiveness of a specific type of computer-based worked example, one designed to encourage students to study the example in an optimal fashion by: (1) incorporating visually isolated and labeled subgoals, a structural manipulation that appears to enhance the way in which students study examples; as well as (2) presenting problem states sequentially, a manipulation that appears to have the potential to accomplish the same goal. The study also examined the effects of having examples present or absent during practice problem solving. Findings indicated that sequentially-presented examples with clearly isolated subgoals produce better conceptual performance than do examples in which solutions are presented all at once without strong subgoal emphasis. It is still unclear whether examples should be present or withdrawn during practice problem solving.
\end{abstract}

Keywords: learning environments, mathematics education, cognitive science

\section{Introduction}

One advantage of using the computer as a means for delivering instruction is that it enables us to combine multiple instructional principles or components in an instructional example. Previous research indicated that several structural manipulations may enhance example processing and offer substantial learning gains. For instance, by sequentially displaying problem states, an example could be designed so that it would focus the student $=\mathrm{s}$ attention on the process of constructing a solution to a problem, allowing the student to examine each component of the example $=\mathrm{s}$ solution in relative isolation from the one preceding it. That is, instead of appearing on the screen as a completely worked problem, as is the case with examples that simultaneously display all of the solution components ("simultaneous examples"), the sequential example appears initially unsolved. The learner then moves forward through the example and watches as problem states are successively added over a series of pages, similar to an animation, with the final page in the series representing the solution in its entirety. This feature may encourage students to engage in anticipative reasoning (demonstrated to be a successful example-processing style) by allowing students to anticipate the next step in an example $=\mathrm{s}$ solution (Renkl, 1997). Moreover, based on the research by Mousavi, Low, and Sweller (1995), a student could hear instructive text while visually inspecting related diagrammatic information presented on the screen, which may effectively enlarge his or her working-memory capacity, thereby increasing the learner=s ability to grasp the information presented in the example. Finally, in order to leverage the advantages of subgoal-oriented instruction (Catrambone, 1998), the example=s solution could be constructed so that it is segmented to correspond to the problem subgoals. In the present study, the type of example, sequential /subgoal-oriented (SE/SO) and simultaneous/non-subgoaloriented (SI/NS), as well as the availability of worked examples during problem solving in both present and absent conditions, was experimentally manipulated. The performances of learners exposed to these experimental manipulations were compared for differences on a variety of outcome measures.

\section{Method}

One hundred and ninety-eight participants were drawn from several undergraduate courses offered at a small, northeastern college and they were randomly assigned to one of four conditions. (1) SE/SO-present ( $n=48)$. The examples in this condition were sequential and contained subgoal cues. The sequential aspect was implemented as follows: The SE/SO example appeared initially unsolved. The user then was able to listen to a tutor=s voice highlight each solution step as each step was visually constructed over a series of frames. The final frame depicted the completely 
worked-out problem. SE/SO examples also contained two explicit cues designed to demarcate a problem=s subgoals; that is, each subgoal was visually isolated and labeled. (2) SE/SO-absent ( $n=49)$. The participants in this condition were not able to view the SE/SO worked examples during practice problem solving. (3) SI/NS-present ( $n=51$ ). Participants in this condition were provided with worked examples in which a voice-over explained the solution steps that were represented on the screen in a simultaneous fashion. Moreover, the SI/NS solutions were lacking two visually explicit subgoal segmentation cues found in the SE/SO examples and were not present during practice problem solving. (4) SI/NS-absent $(n=50)$. The participants in this condition were not able to view the SI/NS worked examples during practice problem solving. This study was conducted in two sessions: (1) a review and pretest session, and (2) an instructional and posttest session. During instruction, participants were exposed to four sets of multi-step proportion word problems, with each set consisting of a condition-specific example along with a paired practice problem.

\section{Results and Discussion}

This study examined three comparisons. First, it was anticipated that the SE/SO students would outperform their SI/NS counterparts on several measures of performance. As predicted, there was a statistically significant difference, $t(186)=1.67, p<.05,{ }^{2}=.01$, with the posttest problem-solving protocols of the SE/SO participants containing evidence of a higher degree of conceptual knowledge than their SI/NS peers. Moreover, participants assigned to the $\mathrm{SE} / \mathrm{SO}$ condition reported understanding the four examples significantly more often than their SI/NS peers, $t(186)=$ $2.19, p<.05,{ }_{-}{ }^{2}=.02$. The second comparison examined differences for SE/SO participants when example availability was manipulated (i.e., present versus absent). Participants assigned to the SE/SO-absent condition reported understanding the examples significantly better than those assigned to the SE/SO-present condition, $t(186)=-4.15, p<.05,{ }_{-}{ }^{2}=.14$. An analysis of conceptual scores on practice problems, however, revealed that SE/SO participants who had an example present during problem solving had more conceptually accurate solutions to the practice problem than those participants not permitted to view the example, $t(186)=2.69, p<.05,{ }^{2}=.06$. Also, a significant difference was found on posttest processing time, $t(186)=-2.20, p<.05,{ }_{-}{ }^{2}=.04$, in which participants in the SE/SO-absent condition took longer to complete the posttest than did the SE/SO-present participants. A third set of comparisons examined whether the presence or absence of examples during problem solving would systematically impact participants= performance in the context of the more conventionallyformatted SI/NS examples. Similar to findings with the SE/SO examples, the SI/NSabsent participants reported that they were better able to understand the examples than were their SI/NS peers who had the example available while solving practice problems, $t(186)=-7.36, p<.05,{ }^{2}=.35$.

In summary, the present study provides additional support for the claim that sequential, subgoal-oriented examples are superior to conventional, simultaneous examples that lack subgoal-oriented instruction. Although the benefits did not manifest themselves on all measures used in this study, students in the SE/SO example condition did outperform those in the SI/NS condition on a conceptually-based scoring of the posttest, and participants in the former condition reported understanding the examples better than did their latter counterparts. This study also produced some evidence that the performance of the participants in the examples-present conditions was superior to the performance of the participants in the absent conditions, on measures of conceptual understanding during problem-solving practice, as well as posttest processing time. But the absent participantsthought they had better understanding of the examples. Thus, the present findings appear not to provide any definitive answers as to whether learning is facilitated by having examples present or absent during problem solving.

\section{Endnotes}

${ }^{1}$ This research was supported by the Office of Naval Research, Cognitive and Neural Sciences Division, under Grant N0001495PR34F2 awarded to Sharon J. Derry.

\section{References}

Catrambone, R. (1998). The subgoal learning model: Creating better examples so that students can solve novel problems. Journal of Experimental Psychology: General, 127, 355-376.

Chi, M. T., Bassok, M., Lewis, M. W., Reimann, P., \& Glaser, R. (1989). Self-explanations: How students study and use examples in learning to solve problems. Cognitive Science, 13, 145-182.

Mousavi, S. Y., Low, R., \& Sweller, J. (1995). Reducing cognitive load by mixing auditory and visual presentation modes. Journal of Educational Psychology, 87, 319-334.

Renkl, A. (1997). Learning from worked-out examples: A study on individual differences. Cognitive Science, 21, 1-29. 